\title{
Targeting Insulin-Like Growth Factor-I receptor signaling pathways improve compromised function during cardiac hypertrophy
}

\author{
Subhalakshmi Ganguly, Emeli Chatterjee, and Sagartirtha Sarkar* \\ Department of Zoology,University of Calcutta, Kolkata, West Bengal, India
}

\begin{abstract}
Insulin-like growth factor-I receptor (IGF-IR) and its signaling pathways play an important role in cell proliferation, growth, differentiation and survival. IGF-axis controls cellular, physiological and metabolic activity of several cell types including cardiomyocytes. IGF-I acts through its signaling receptor- IGF-IR to induce physiological or adaptive hypertrophic growth. Various reports also confirm that it protects the heart against pathological insults. Furthermore, deregulation of IGF-I signaling has also been shown to be involved in the development of pathological hypertrophy. Although it would be beneficial to activate the IGF-I-PI3-K pathway to enhance cardiac function during pathological conditions it could be detrimental in case of cancer. Based on currently available literature this mini review will focus on the recent advances of drug and delivery systems that specifically target IGF-I signaling for promoting physiological hypertrophy and improving cardiac function.
\end{abstract}

\begin{abstract}
Abbreviations: Insulin-like growth factor-I (IGF-I); Insulin-like growth factor-I receptor (IGF-IR); Mammalian target of rapamycin (mTOR); 4E binding protein 1 (4EBP1); local IGF-1 isoform (mIGF-1); (PI3K)-AKT/PKB phosphatidylinositol 3-kinase-Akt/Protein Kinase $\mathrm{B}$; Insulin receptor (IR); MicroRNAs (miRs); Ribosomal S6 kinases (S6Ks)
\end{abstract}

\section{Introduction}

IGF-I, a 70 amino acid single chain protein is synthesized primarily in liver, but the local production in other tissues mediates growthpromoting effects by autocrine or paracrine mechanisms [1,2]. A large number of studies have described the presence and distribution of IGF's and IGF receptors in heart [1-3]. In cardiomyocytes, IGF-I activates multiple downstream signaling pathways for controlling cardiomyocyte survival, autophagy, apoptosis, differentiation, and protein synthesis $[1,2]$. Other diverse physiological roles of this ligand (IGF-I) receptor family in heart include energy balance, metabolism of glucose, carbohydrate and protein $[2,4]$.

Heart growth often referred to as cardiac hypertrophy can broadly be classified as pathological or physiological [5]. Pathological cardiac hypertrophy occurs in response to hypertension, myocardial infarction, valvular heart disease and is associated with accumulation of collagen, depressed cardiac function and heart failure [6,7]. In contrast, physiological hypertrophy includes heart growth in response to chronic exercise training and is associated with preserved or enhanced cardiac function [8,9]. This difference in outcome of pathological and physiological cardiac hypertrophy is due to different stimuli leading to the activation of distinct signaling transducers [5,9-11]

To date, the best characterized pathways playing distinct roles for induction of pathological and physiological hypertrophy are Gaq and insulin-like growth factor (IGF) -I -phosphoinositide-3 kinase (PI3-K; p110a), respectively [5]. Several reports have shown that chronic exercise training such as running, walking, cycling and swimming elevates IGF-I levels and subsequent activation of IGFIR leads to physiological cardiac hypertrophy $[6,12,13]$. Conversely, Ang II, ET-1 and noradrenaline are secreted from cardiomyocytes during mechanical stress and the signal is transduced by binding to $\mathrm{G} q$ protein-coupled receptors (GPCR). Gq mediates pathological cardiac hypertrophy via downstream effectors like calcineurin and (mitogenactivated protein kinases (MAPK); extracellular signal regulated kinases 1 and 2 (ERK1/2), JNK, P38) [5,9].

\section{IGF-I signaling cascade mediated physiological cardiac hypertrophy}

It is now well established that IGF-I plays an important role in the regulation of post-natal heart size and cardiac function $[9,10,14]$. IGF-I is primarily produced in liver and to a lesser extent locally in heart [2]. Exercise induces cardiac expression of both IGF-I and its receptor IGF-IR [13-15]. IGF-I levels are found to be elevated in both swim-trained rats and athlete's heart compared to control subjects [5]. Activation of IGF-IR (receptor tyrosine kinase) leads to physiological cardiac hypertrophy in mice characterized by $35-40 \%$ increase in heart size and enhanced systolic function [2,9]. Again, mice with cardiac specific ablation of the IGF-IR gene are resistant to exercise-induced increases in heart size $[15,16]$. IGF-I in heart via IGF-IR activates

Correspondence to: Department of Zoology, University of Calcutta 35, Ballygunge Circular Road, Kolkata - 700019, West Bengal, India. Tel: +91 33 2475 3681; E-mail: sagartirtha.sarkar@gmail.com

Key words: insulin-like growth factor-I receptor, hypertrophy, pathological, physiological, cardiomyocytes

Received: October 22, 2015; Accepted: November 10, 2015; Published: November 18, 2015 
phosphoinositide kinase-3 - PI3K (p110a) and its downstream target Akt1 (Protein Kinase B). Both PI3K and AKT are identified to be essential for induction of physiological heart growth [5,9]. It has been shown that mice with constitutively active PI3K (110a) exhibit significantly increased heart mass and are also protected from heart failure after pathological stress $[9,17]$. In contrast, mice with cardiac expression of a dominant negative PI3K (110a) respond to pressure overload instead of swimming exercise $[4,14]$. Similarly, Akt1-knockout mice showed a blunted hypertrophic response to swim training but not to pressure overload [18]. Downstream targets of activated Akt are mammalian target of rapamycin (mTOR) which suppresses autophagy and promotes protein synthesis by activating S6K and eukaryotic translation initiation factor $4 \mathrm{E}$ binding protein 1 (4EBP1) $[5,13]$. The activity of S6K1 has been shown to be elevated in transgenic models of physiological hypertrophy [5].

\section{miRs involved in the IGF-I-PI3K-Akt pathway in heart}

MicroRNAs (miRs) are important endogenous post-transcriptional regulators of gene expression involved in regulation of various disease and development processes. Since 2005, lot of exciting research has come up on the biological roles of miRs in the mammalian cardiovascular system [19]. Extensive genome-wide profiling of miRs has shown significant changes in expression of many miRs during physiological, pathological cardiac hypertrophy and heart failure [1921]. Here we have highlighted miRs that are involved in the IGF-IPI3K-Akt signaling pathway in heart.

miR-1, the most abundant of all miRs in heart plays a key role in determining cardiomyocyte morphology and function [20,22]. As evident from luciferase activity Assays, 3'UTR sequence of IGF-I has only one potential MiR-1binding site [20,23]. miRs -1 and -133a have been shown to abrogate IGF-I induced hypertrophy. This suggests requirement for miR-1 and miR-133a downregulation in IGF-I induced physiological hypertrophy [22]. Both of these miR's have been shown to be down-regulated in exercised trained rats and cardiacspecific Akt transgenic mice, which are models of physiological cardiac hypertrophy [24].

Interestingly few reports have shown IGF-I not only is a target of miR-1but miR-1 expression also depends on IGF-I [20,24]. In neonatal cardiomyocytes, IGF-I treatment significantly repressed miR1 expression. IGF-I, through activation of the PI3K/AKT signaling pathway and repression of its downstream transcription factor Foxo3a silences miR-1. Further detailed research on this unique miR-1/IGF-I regulatory loop will help us understand in future the importance of IGF-I-mediated effects on cardiac hypertrophy and heart failure [25].

Another miRNAs which is abundantly induced in heart during stress is miR-378. According to one study, ligand IGF-I acts as an inhibitor of miR-378 expression in heart. The report further showed miR-378 inhibits IGF-I mediated activation of AKT by directly targeting IGF-IR thereby providing resistance to cardiomyocytes against stressmediated cell death. Hence inhibition of miR-378 would perhaps be beneficial for the survival of cardiomyocytes during compromised heart function. However, its effect on cardiac hypertrophy is not yet reported [26].

\section{IGF-I based treatments and challenges}

The importance of the IGF-I pathway in cardiac function is well established $[9,10]$. This largely explains the interest regarding the use of new agents that block pathological hypertrophy in conjunction with IGF-I-based treatments that promote physiological cardiac growth and improve contractile function [14]. IGF-I deficiency is found to be associated with an elevated risk of cardiovascular disease and its progression in both human and animals [1]. So far, mecasermin $\left(\right.$ Increlex ${ }^{\mathrm{R}}$ ); a human recombinant IGF-I analog and mecaserminrinfabate (IPLEX ${ }^{\mathrm{R}}$ ); a binary protein complex of human recombinant IGF-I and human recombinant IGBP-3, are two IGFI-based treatments available for improvement of cardiac function during IGF-I deficiency [1]. Few clinical trials have shown that Growth Hormone (GH) administration leads to improved cardiac performance in patients with impaired cardiac function [2]. It has been further confirmed that patients whose IGF-I level fails to increase in response to exogenous $\mathrm{GH}$ administration do not benefit from $\mathrm{GH}$ treatment. Results further revealed that IGF-I level increased by $>89 \%$ would significantly improve cardiac performance, echocardiographic parameters and exercise capacity in patients compared to patients with IGF-I increase by $<89 \%$ following exogenous GH administration [2]. Few reports have clearly shown that persistent local IGF-I expression in the heart results in physiological cardiac hypertrophy at early time points [14,27]. Also, short term administration of IGF-I is beneficial in improving cardiac function [14]. Transgenic mice overexpressing IGF-I receptors (IGF-IR) in cardiomyocytes also shows physiological growth of the heart [14]. In addition to induction of physiological hypertrophy, IGF-I-PI3-K pathway is also activated in some pathological models as a protective mechanism [5]. In the recent years, evidences have been mounting on the attempts made to selectively overexpress IGF-I and IGF-IR in the heart. One research group showed that local IGF-I isoform (mIGF-I) protects cardiomyocytes from hypertrophic stress. It was further shown that potent cardio-protective genes are upregulated specifically in mIGF-1-overexpressing cardiomyocytes. Based on these observations it could be postulated that mIGF-1 might be a promising cardiac therapeutic against hypertrophic stress [27].

Several biological processes controlling cardiac function are regulated by miRNA's and IGF-I. Hence miRNA's mediating the effects of IGF-I pathway could serve as potential therapeutic agents for cardiac hypertrophy $[24,28,29]$.

IGF-I/IGF-IR pathway is well recognized for its role in various cancers [30]. Although activation of IGF1-PI3K (p110a) pathway by chronic systemic IGF-I therapy, miRs's or miRs-targeting oligonucleotides, would be beneficial for heart it could lead to uncontrolled proliferation of other cell types ultimately leading to cancer $[10,30]$. Again, excess of IGF-I signaling may also trigger cardiac dysfunction [24]. Hence local versus systemic effects of IGF-I administration must be elaborately evaluated [31]. More works are also needed to address the issues associated with the outcomes of quantitative versus qualitative differences in exposure of pathological and physiological stimuli [4]. Furthermore, it has been shown Insulin receptor (IR) and IGF-IR are structurally similar and once activated can phosphorylate same downstream signaling. Reports are also available about Hybrid receptors that are formed between IGF-IR and IR in several tissues [16]. Although it is known that IGF-IR and not IR signaling is an important regulator of exercise-induced physiological cardiac hypertrophy yet more studies are required to understand 
the effect of cardiac specific IGF-I/IGF-IR overexpression on IR and IGF-IR/IR- hybrid receptors $[15,16]$. IGF-I is known to be a marker in cancer and other diseases. But in spite of being elevated, it is not considered to be a good biomarker for the athlete's heart $[1,9,12]$. Despite few reports on targeted delivery of oligonucleotides, poor bioavailability, limited tissue permeability, potential drug resistance and instability still remain major challenges till date [28]. Recently, one report on a novel cardiomyocyte targeted nano-construct promises to be a potential clinical tool and provide a solution to some of these challenges [32].

\section{Conclusion}

Cardiac hypertrophy is one of the important cardiovascular problems throughout the world [6]. Substantial progress has been made in the understanding of signaling pathways mediating physiological and pathological hypertrophy. While exercise induces physiological heart growth mechanical stress due to pressure overload leads to pathological hypertrophy [9]. Pathological hypertrophic characters are also evident after withdrawal from regular exercise training [33]. It would be interesting to address in future whether IGF-IR pathway plays any crucial role in the onset of pathological hypertrophy after withdrawal from exercise training. Exercise induces physiological heart growth via activation if IGF-I-PI3-K pathway [9]. Since many patients are unable to exercise, therefore understanding of the pathways that mediate exercise induced benefits, learning to manipulate them in vivo and providing solution to existing challenges as discussed could yield novel therapeutic approaches to improve cardiac function during pathological hypertrophy [4].

\section{Conflict of interest}

The author(s) confirm that this article content has no conflict of interest.

\section{Acknowledgements}

This work was funded by grants from Department of Biotechnology, Government of India (BT/PR3709/BRB/10/980/2011) and Department of Science and Technology Government of India (SR/SO/HS-100/2009) to S.S. Dr. S.Ganguly was supported by Dr. D. S. Kothari post doctoral fellowship scheme [No.F.4-2/2006 (BSR)/BL/13-14/0289] from University Grants Commission, Government of India.

\section{References}

1. Troncoso R, Ibarra C, Vicencio JM, Jaimovich E, Lavandero S, et al. (2014) New insights into IGF-1 signaling in the heart. Trends Endocrinol Metab 25: 128-137.

2. Arcopinto M, Bobbio E, Bossone E, Perrone-Filardi P, Napoli R, et al. (2013) The GH/ IGF-1 Axis and Heart Failure. Endocr Metab Immune Disord Drug Targets 13: 76-91.

3. Fazio S, Palmieri EA, Biondi B, Cittadini A, Saccà L (2000) The role of the GHIGF-1 axis in the regulation of myocardial growth: from experimental model to human evidence. Eur J Endocrinol 142: 211-216.

4. Mann N, Rosenzweig A (2012) Can exercise teach us how to treat heart disease? Circulation 126: 2625-2635. [Crossref]

5. McMullen JR, Jennings GL (2007) Differences between pathological and physiological cardiac hypertrophy: novel therapeutic strategies to treat heart failure. Clin Exp Pharmacol Physiol 34: 255-262.

6. Rohilla A, Kumar P, Rohilla S, Kushnoor A (2012) Cardiac Hypertrophy: A Review on Pathogenesis and Treatment. Int of Pharmac Sci Drug Res 4: 164-167.

7. Iemitsu M, Miyauchi T, Maeda S, Sakai S, Kobayashi T, et al. (2001) Physiological and pathological cardiac hypertrophy induce different molecular phenotypes in the rat. AJP - Regu Physiol 281: 2029-2036.
8. Mone SM, Sanders SP, Colan SD (1996) Control mechanisms for physiological hypertrophy of pregnancy. Circulation 94: 667-672.

9. Weeks KL, McMullen JR (2011) The athlete's heart vs. the failing heart: can signaling explain the two distinct outcomes? Physiology 26: 97-105. [Crossref]

10. McMullen JR (2008) Role of insulin-like growth factorl and phosphoinositide 3-kinase in a setting of heart disease. Clin Exp Pharmacol and Physiol 35: 349-354.

11. Carreño JE, Apablaza F, Ocaranza MP, Jalil JE (2006) Cardiac hypertrophy: molecular and cellular events. Rev Esp Cardio 59: 473-86. [Crossref]

12. NeriSerneri GG, Boddi M, Modesti PA, Cecioni I, Coppo M, et al. (2001) Increased cardiac sympathetic activity and insulin-like growth factor-I formation are associated with physiological hypertrophy in athletes. Circ Res 89: 977-982. [Crossref]

13. Rohini A, Agrawal N, Koyani CN, Singh R (2010) Molecular targets and regulators of cardiac hypertrophy. Pharmacol Res 61: 269-80. [Crossref]

14. McMullen JR, Shioi T, Huang WY, Zhang L, Tarnavski O, et al. (2004) The insulin-like growth factor 1 receptor induces physiological heart growth via the phosphoinositide 3-Kinase(p110) Pathway. J Biol Chem 279: 4782-4793. [Crossref]

15. Kim J, Wende AR, Sena S, Theobald HA, Soto J, et al. (2008) Insulin-like growth factor i receptor signaling is required for exercise-induced cardiac hypertrophy. Mol Endocrinol 22: 2531-2543. [Crossref]

16. Laustsen PG, Russell SJ, Cui L, Entingh-Pearsall A, Holzenberger M, et al. (2007) Essential role of insulin and insulin-like growth factor 1 receptor signaling in cardiac development and function. Mol Cell Biol 27: 1649-1664. [Crossref]

17. Matsui T, Nagoshi T, Rosenzweig A (2003) Akt and PI 3-kinase signaling in cardiomyocyte hypertrophy and survival. Cell Cycle 2: 220 -223.

18. DeBosch B, Treskov I, Lupu TS, Weinheimer C, Kovacs A, et al. (2006)Akt1 is required for physiological cardiac growth. Circulation 113: 2097-2104. [Crossref]

19. Wang N, Zhou Z, Liao X, Zhang T (2009) Role of microRNAs in cardiachypertrophy and heartfailure. IUBMB Life 61: 566-571. [Crossref]

20. Gladka MM, da Costa Martins PA, De Windt LJ (2012) Small changes can make a big difference - microRNA regulation of cardiac hypertrophy. J Mol Cell Cardiol 52: 74-82. [Crossref]

21. Ikeda S, Kong SW, Lu J, Bisping E, Zhang H, et al. (2007) Altered microRNA expression in human heart disease. Physiol Genomics 31: 367-373. [Crossref]

22. Winbanks CE, Ooi JY, Nguyen SS, McMullen JR, Bernardo BC (2014) MicroRNAs differentially regulated in cardiac and skeletal muscle in health and disease: potential drug targets? Clin Exp Pharmacol Physiol 41: 727-737. [Crossref]

23. Elia L, Contu R, Quintavalle M, VarroneF, ChimentiC, etal. (2009) Reciprocalregulation of microRNA-1 and insulin-like growth factor-1 signal transduction cascade in cardiac and skeletal muscle in physiological and pathological conditions. Circulation 120 : 2377-2385. [Crossref]

24. Wang J, Yang X (2012) The function of miRNA in cardiac hypertrophy. Cell Mol Life Sci 69: 3561-3570.

25. Bauersachs J, Thum T (2011) Biogenesis and regulation of cardiovascular microRNAs Circ Res 109: 334-347. [Crossref]

26. Knezevic I, Patel A, Sundaresan NR, Gupta MP, Solaro RJ, et al. (2012) A nove cardiomyocyte-enriched microRNA, miR-378, targets insulin-like growth factor 1 receptor: implications in postnatal cardiac remodeling and cell survival. $J$ BiolChem 287: 12913-12926. [Crossref]

27. Vinciguerra M, Santini MP, Claycomb WC, Ladurner AG, Rosenthal N (2009) Loca IGF-1 isoform protects cardiomyocytes from hypertrophic and oxidative stresses via SIRT1 activity. Aging (Albany NY) 2: 43-62. [Crossref]

28. Jung HJ, Suh Y (2015) Regulation of IGF -1 signaling by microRNAs. Front Genet 5: 472

29. Elia L, Contu R, Quintavalle M, Varrone F, Chimenti C, et al. (2009) Reciprocal regulation of microRNA-1 and insulin-like growth factor-1 signal transduction cascade in cardiac and skeletal muscle in physiological and pathological conditions. Circulation 120: 2377-2385. [Crossref]

30. Ganguly S, Basu B, Shome S, Jadhav T, Roy S, et al. (2010) Dopamine, by Acting through Its D2 Receptor, Inhibits Insulin-Like Growth Factor-I (IGF-I)-Induced Gastric Cancer Cell Proliferation via Up-Regulation of Krüppel-Like Factor 4 through Down-Regulation of IGF-IR and AKT Phosphorylation. Am J Pathol 177: 2701-2707.

31. Samarel AM (2002) IGF-1 Overexpression rescues the failing heart. Circ Res 90 $631-3$ 
Ganguly S (2015) Targeting Insulin-Like Growth Factor-I receptor signaling pathways improve compromised function during cardiac hypertrophy

32. Rana S, Datta K, Reddy TL, Chatterjee E, Sen P, et al. (2015) Spatio-temporal cardiomyocyte targeted vector system for efficient delivery of therapeutic payloads to regress cardiac hypertrophy abating bystander effect. $J$ Control Release 167-178. [Crossref]
33. Naskar S, Datta K, Mitra A, Pathak K, Datta R, et al. (2014) Differential and conditional activation of $\mathrm{PKC}$-isoforms dictates cardiac adaptation during physiological to pathological hypertrophy. PLoS One 9: e104711.

Copyright: $\odot 2015$ Ganguly S. This is an open-access article distributed under the terms of the Creative Commons Attribution License, which permits unrestricted use, distribution, and reproduction in any medium, provided the original author and source are credited. 